\title{
Correction to: Genetic polymorphisms and mutation rates of 16 X-STRs in a Han Chinese population of Beijing and application examples in second-degree kinship cases
}

\author{
Man Chen ${ }^{1,2} \cdot$ He Ren $^{3} \cdot$ Zhiyong Liu ${ }^{1,2} \cdot$ Jing Zhao ${ }^{1} \cdot$ Chong Chen ${ }^{4} \cdot$ Yan Shi ${ }^{4} \cdot$ Li Jia $^{4} \cdot$ Feng Cheng $^{5} \cdot$ Tong Chen $^{1,2}$. \\ Qingwei Fan ${ }^{5}$ - Yaran Yang ${ }^{1}$. Yacheng Liu ${ }^{4}$. Gengqian Zhang ${ }^{5}$. Jiangwei Yan ${ }^{1,2,5}$ (D)
}

Published online: 22 April 2019

(C) Springer-Verlag GmbH Germany, part of Springer Nature 2019

Correction to: International Journal of Legal Medicine 2019

https://doi.org/10.1007/s00414-019-02047-8

The above article was published online with incorrect author name. The right spelling should be Gengqian Zhang instead of Genqian Zhang. The original article has been corrected.

Publisher's note Springer Nature remains neutral with regard to jurisdictional claims in published maps and institutional affiliations.

The online version of the original article can be found at https://doi.org/ 10.1007/s00414-019-02047-8

Gengqian Zhang

gengqianzhang@sxmu.edu.cn

$\triangle$ Jiangwei Yan

yanjw@big.ac.cn

1 CAS Key Laboratory of Genome Sciences and Information, Beijing Institute of Genomics, Chinese Academy of Sciences,

Beijing 100101, People's Republic of China

2 University of Chinese Academy of Sciences, Beijing 100049, People's Republic of China

3 Beijing Police College, Beijing 102202, People's Republic of China

4 Beijing Tongda Shoucheng Institute of Forensic Science, Beijing 100192, People's Republic of China

5 School of Forensic Medicine, Shanxi Medical University, Taiyuan 030009, People's Republic of China 